\title{
Event Shapes and Power Corrections in ep DIS
}

\author{
K. Rabbertz ${ }^{\mathrm{a}}$ \\ ${ }^{a}$ I. Physikalisches Institut, RWTH Aachen, D-52056 Aachen, Germany \\ (On behalf of the H1 Collaboration)
}

Deep-inelastic ep scattering data, taken with the H1 detector at HERA, are used to study the event shape variables thrust, jet broadening, jet mass, $C$ parameter, and two kinds of differential two-jet rates over a large range of "relevant energy" $Q$ between $7 \mathrm{GeV}$ and $100 \mathrm{GeV}$.

The $Q$ dependence of the mean values is fit to second order calculations of perturbative QCD applying power law corrections proportional to $1 / Q^{p}$ to account for hadronization effects. The concept of these power corrections is tested by a systematic investigation in terms of a non-perturbative parameter $\bar{\alpha}_{p-1}$ and the strong coupling constant.

\section{INTRODUCTION}

Due to asymptotic freedom perturbative QCD is a powerful theoretical tool for the investigation of strong interactions at high energies $Q$. Given, however, the precision of today's measurements over a large range of energy, deviations diminishing proportional to $1 / Q^{p}$ are observed. Depending on the sensitivity of the observable on long-distance, non-perturbative effects, these power corrections may be sizable. Less inclusive quantities than e.g. the total cross section of $e^{+} e^{-} \rightarrow$ hadrons, where $p=4$, exhibit much larger corrections; in case of event shapes they are typically proportional to $1 / Q$.

One possibility to account for the nonperturbative formation of hadrons out of quarks and gluons is to invoke phenomenological models implemented in Monte Carlo generators. As a drawback, one has to deal with several models, each again comprising a large number of tunable parameters. Alternatively, analytical formulae either inspired by a simple tube model [ [1] or by a recent approach initiated by Dokshitzer and Webber [2] may be employed. A first study of event shapes and analytically parameterized power corrections in $e p$ deep-inelastic scattering (DIS) was published in [ [3]. The results presented here are based on the substantially improved and extended analysis in [四].

\section{EVENT SHAPE VARIABLES}

The event shapes investigated are two kinds of 1-thrust, $\tau$ and $\tau_{C}$, the jet broadening $B$, the jet mass $\rho$, the $C$ parameter, and two kinds of differential two-jet rates, $y_{f J}$ and $y_{k_{t}}$, with $y$ denoting the transition value of an event from $(2+1) \rightarrow(1+1)$ jets. All of these infrared and collinear safe variables are defined in the Breit frame of reference. Except for the two jet observables $y_{f J}$ and $y_{k_{t}}$, they exploit the property of this frame to maximally separate the current quark jet from the proton remnant by evaluating the current hemisphere alone. They are normalized to the total momentum resp. the total energy in the current hemisphere; the precise definitions may be found in [ 田, 可. In case of the $y$ variables, the employed jet algorithms, i.e. the factorizable JADE and the $k_{t}$ algorithm [6], implicitly isolate the proton remnant.

The energy scale relevant in DIS is given by the four-momentum transfer $q$ of the scattered lepton as $Q:=\sqrt{-q^{2}}$ and ranges from $7 \mathrm{GeV}$ up to $100 \mathrm{GeV}$ in this study. Note that a second quantity, e.g. the Bjørken scaling variable $x:=-q^{2} / 2 P \cdot q$ with $P$ being the fourmomentum of the incoming proton, is necessary to fix the DIS kinematics. Here, $x$ varies between $\approx 10^{-3}$ and $\approx 0.5$. 


\section{POWER CORRECTIONS}

According to [2] the energy dependence of any event shape mean $\langle F\rangle$ can be written as sum of a perturbative part $\langle F\rangle^{\text {pert }}$ and a power suppressed contribution $\langle F\rangle^{\text {pow }}$. Identifying for simplicity the renormalization scale $\mu_{R}$ with $Q$, the perturbative part, evaluated to $\mathcal{O}\left(\alpha_{s}^{2}\right)$ using DISENT [ 7], is given by

$$
\langle F\rangle^{\text {pert }}=c_{1, F}(x) \alpha_{s}(Q)+c_{2, F}(x) \alpha_{s}^{2}(Q) .
$$

Note that in contrast to $e^{+} e^{-}$annihilation parton density functions are necessary to describe the initial state in $e p$ DIS. Apart from other quantities the coefficients therefore explicitly depend on $x$.

Anticipating power corrections proportional to $1 / Q$ and $1 / Q^{2}$ in the case of $\left\langle y_{k_{t}}\right\rangle$, the simple ansatz of $\langle F\rangle^{\text {pow }}=\lambda / Q$ resp. $\langle F\rangle^{\text {pow }}=\mu / Q^{2}$ is tested. However, fits of $\lambda$ or $\mu$ alone are not able to describe the data satisfactorily. Fitting also $\alpha_{s}\left(M_{Z}\right)$ mostly leads to unacceptable values of $\alpha_{s}\left(M_{Z}\right)$ owing to strong correlations. Triggered by the $x$ dependence of $\langle F\rangle^{\text {pert }}$, one could assume that $\langle F\rangle^{\text {pow }}$ should be a function of $x$ as well. Yet, for corrections with power $p=1$ this is not expected [8].

This calls for the more elaborate approach of [ 2], referring infrared divergences in the perturbative expansion to the power corrections. Assuming the existence of a well-behaved effective strong coupling even at scales below the infrared matching scale $\mu_{I}$ of $\approx 2 \mathrm{GeV}$, one can deduce the relative size of the corrections $\mathcal{P} / Q$ to the means of different event shapes at the expense of a new non-perturbative parameter $\bar{\alpha}_{0}$ :

$$
\begin{aligned}
\mathcal{P}= & a_{F} \mathcal{M}^{\prime} \frac{16 \mu_{I}}{3 \pi}\left[\bar{\alpha}_{0}\left(\mu_{I}\right)-\alpha_{s}(Q)\right. \\
& \left.-\frac{23}{6 \pi}\left(\ln \frac{Q}{\mu_{I}}+1.45\right) \alpha_{s}^{2}(Q)\right],
\end{aligned}
$$

where $a_{F}$ is a calculable $F$ dependent coefficient. To resolve ambiguities a refinement to two-loop level leads to another global factor, the Milan factor $\mathcal{M}$, where $\mathcal{M}^{\prime}=2 \mathcal{M} / \pi \approx 1.14$ [ 9 ].

As an example, fig. 1 shows the unfolded differential distributions and a power correction fit for $y_{f J}$, however, with $a_{y_{f J}}=-1 / 4$.
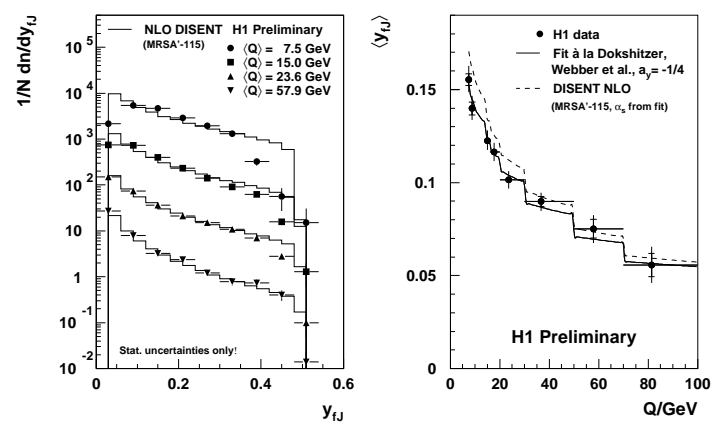

Figure 1. Left: Norm. diff. distr. of $y_{f J}$ corrected for detector effects. H1 data (stat. uncert. only) are compared with NLO calculations. Right: Corr. means of $y_{f J}$ (stat. and tot. uncert.) as a function of $Q$. The full line corresponds to a power correction fit with $a_{y_{f J}}=-1 / 4$.

The results of the two-parameter fits to this approach are presented in table 1 and fig. 2. The universal non-perturbative parameter $\bar{\alpha}_{0}\left(\mu_{I}=\right.$ $2 \mathrm{GeV}$ ) is confirmed to be $\approx 0.5 \pm 20 \%$ for the means of $\tau, B, \tau_{C}, \rho$, and $C$, which are sizably affected by hadronization. The spread in $\alpha_{s}\left(M_{Z}\right)$, however, is uncomfortably large. In contrast to earlier analyses of $B$, a reasonable fit is obtained if the revised coefficient $a_{B}^{\prime}$ [ [10] is included.

Both $y$ observables exhibit small negative hadronization corrections. For $y_{f J}$ a power correction with $p=1$ and $a_{y_{f J}}=1$ was proposed in the conference proceedings [ 11], which has not been reconsidered in recent publications. From the emerging very low values for both, $\bar{\alpha}_{0}\left(\mu_{I}=\right.$ $2 \mathrm{GeV})$ and $\alpha_{s}\left(M_{Z}\right)$, one can rule out $a_{y_{f J}}=1$. Turning the argument around, a reasonable fit can be achieved with $a_{y_{f J}}=-1 / 4$ while presupposing the validity of eq. 2. For $y_{k_{t}}$ fits with $p=1$ do not work properly in accordance with the expectation of a power $p=2$. Unfortunately, the coefficients $a_{F}$ are not yet calculable for that case.

\section{SUMMARY AND CONCLUSIONS}

The improved and extended analysis of event shape means in ep DIS supports the concept of power corrections according to eq. 2. However, the uncomfortably large spread of values gained for $\alpha_{s}\left(M_{Z}\right)$ leaves room for further progress. 
Table 1

Results of two-parameter fits of $\bar{\alpha}_{0}\left(\mu_{I}=2 \mathrm{GeV}\right)$ and $\alpha_{s}\left(M_{Z}\right)$ according to the power correction approach initiated by Dokshitzer and Webber for the event shape means. The uncertainties are statistical and total systematic except for $y_{f J}$ where a coefficient $a_{y_{f J}}$ different from 1 is anticipated. $\kappa$ denotes the correlation coefficient between the two fit parameters.

\begin{tabular}{cccccc}
\hline \multicolumn{5}{c}{ H1 Preliminary } \\
\hline$\langle F\rangle$ & $a_{F}$ & $\bar{\alpha}_{0}\left(\mu_{I}=2 \mathrm{GeV}\right)$ & $\alpha_{s}\left(M_{Z}\right)$ & $\chi^{2} / n$ & $\kappa / \%$ \\
\hline$\langle\tau\rangle$ & 1 & $0.480 \pm 0.028_{-0.062}^{+0.048}$ & $0.1174 \pm 0.0030_{-0.0081}^{+0.0097}$ & 0.5 & -97 \\
$\langle B\rangle$ & $1 / 2 \cdot a_{B}^{\prime}$ & $0.491 \pm 0.005_{-0.036}^{+0.032}$ & $0.1106 \pm 0.0012_{-0.0057}^{+0.0060}$ & 0.7 & -58 \\
$\left\langle\tau_{C}\right\rangle$ & 1 & $0.475 \pm 0.003_{-0.048}^{+0.044}$ & $0.1284 \pm 0.0014_{-0.0092}^{+0.0100}$ & 1.3 & +19 \\
$\langle\rho\rangle$ & $1 / 2$ & $0.561 \pm 0.004_{-0.058}^{+0.051}$ & $0.1347 \pm 0.0015_{-0.0100}^{+0.0111}$ & 1.2 & +7 \\
$\langle C\rangle$ & $3 \pi / 2$ & $0.425 \pm 0.002_{-0.039}^{+0.033}$ & $0.1273 \pm 0.0009_{-0.0093}^{+0.0104}$ & 0.9 & +63 \\
$\left\langle y_{f J}\right\rangle$ & 1 & $0.258 \pm 0.004$ & $0.104 \pm 0.002^{\prime}$ & 1.9 & -61 \\
\hline$a_{B}^{\prime}$ & 1 & $1 / \sqrt{\alpha_{s}}$
\end{tabular}

$a_{B}^{\prime} \propto 1 / \sqrt{\alpha_{s}}+$ const. as suggested by Dokshitzer, Marchesini and Salam [10].

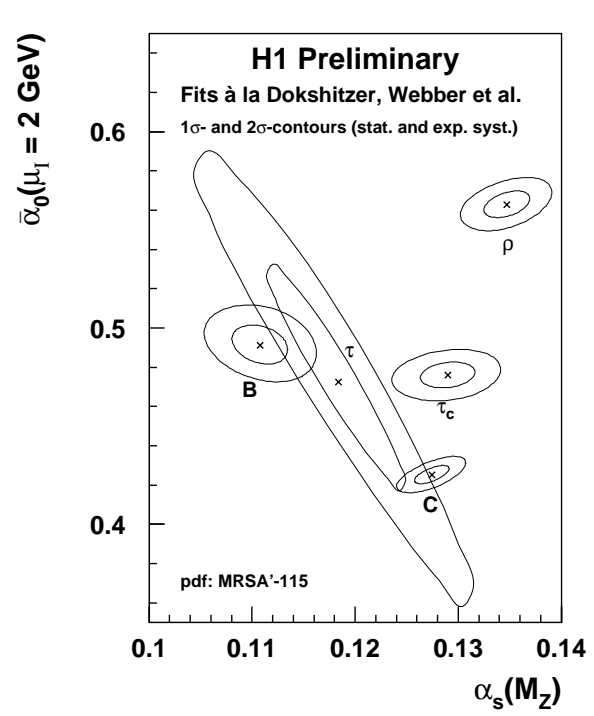

Figure 2. Results of two-parameter power correction fits for the means of $\tau, B, \tau_{C}, \rho$, and $C$ in the form of $1 \sigma$-and $2 \sigma$-contours in the $\left(\alpha_{s}, \bar{\alpha}_{0}\right)$-plane including stat. and exp. syst. uncertainties.

Like in $e^{+} e^{-}$annihilation, it would be very interesting to compare to the power corrections obtained with event shape distributions. To that goal, resummed calculations are awaited for.

\section{ACKNOWLEDGEMENTS}

I would like to thank M. Dasgupta and G.P. Salam for enlightening discussions.

\section{REFERENCES}

1. R.P. Feynman, Photon Hadron Interactions, W.A. Benjamin, New York (1972).

2. Yu.L. Dokshitzer, B.R. Webber, Phys. Lett. B 352 (1995) 451.

3. H1 Collaboration, C. Adloff et al., Phys. Lett. B 406 (1997) 256.

4. K. Rabbertz, PITHA 98/44, PhD thesis, RWTH Aachen (1998), to be published.

5. H.-U. Martyn, in Proc. DIS 98, Brussels, 1998.

6. B.R. Webber, J. Phys. G 19 (1993) 1567; S. Catani, Yu.L. Dokshitzer, B.R. Webber, Phys. Lett. B 285 (1992) 291.

7. S. Catani, M.H. Seymour, Nucl. Phys. B 485 (1997) 291.

8. M. Dasgupta, private communication.

9. Yu.L. Dokshitzer, A. Lucenti, G. Marchesini, G.P. Salam, JHEP 05 (1998) 003; M. Dasgupta, B.R. Webber, hep-ph/9809247.

10. Yu.L. Dokshitzer, G. Marchesini, G.P. Salam, hep-ph/9812487.

11. B.R. Webber, in Proc. DIS 95, Paris, 1995. 